
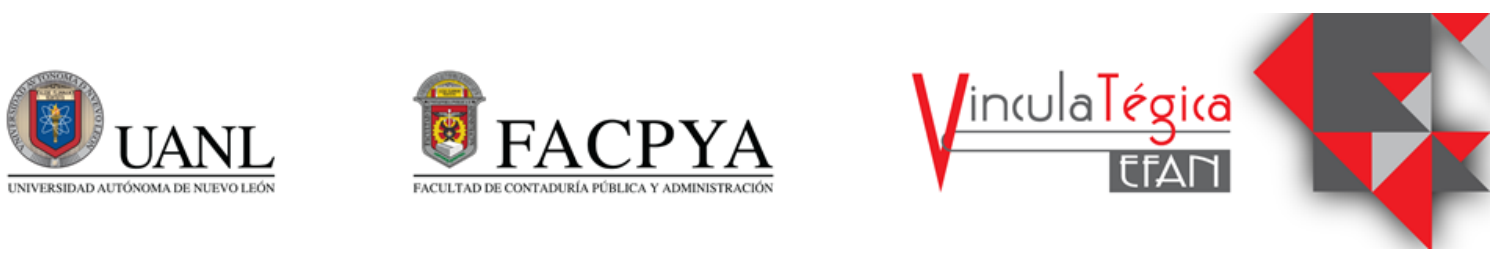

\title{
Revisión sistemática de la literatura: desarrollo de proveedores, innovación y competitividad
}

\author{
Paulina Villalobos Torres ${ }^{1}$ y Luis Ramón Moreno Moreno ${ }^{2}$ \\ ${ }^{1}$ Universidad Autónoma de Baja California , paulina.villalobos@uabc.edu.mx, Blvd. Río Nuevo y Eje Central \\ S/N CP. 21330, 6861642178. \\ ${ }^{2}$ Universidad Autónoma de Baja California, Imoreno@uabc.edu.mx, Blvd. Río Nuevo y Eje Central S/N CP.
} 21330, 6861741165.

Información del artículo revisado por pares

Fecha de aceptación: junio-2021

Fecha de publicación en línea: diciembre-2021

DOI: https://doi.org/10.29105/vtga7.1-146

\section{Resumen}

En la actualidad, las empresas manufactureras se han centrado en la administración de la cadena de suministro y cómo mejorar su competitividad. Las ventajas competitivas son más fuertes si se trabaja en colaboración con los proveedores. En este contexto se analiza la importancia de investigar la relación del desarrollo de proveedores con la mejora de la capacidad competitiva a través de la innovación. El objetivo de este artículo es realizar una revisión sistemática de la literatura relacionada con el desarrollo de proveedores y la competitividad, en particular, la innovación como una ventaja competitiva en ambientes industriales o de manufactura. Los resultados se obtuvieron de artículos científicos recuperados de las bases de datos Ebsco, Scopus, Web of science y Google Académico que fueron publicados entre 2017 y 2021. Se siguió un proceso de selección con la metodología PRISMA. Los resultados demostraron que la tendencia ha ido en incremento en los últimos 5 años principalmente en países desarrollados. Se observa que México tiene una mínima participación en estas investigaciones.

Palabras clave: cadena de suministro, competitividad, desarrollo de proveedores, innovación.
Abstract
Today, manufacturing companies have focused on supply chain management and how to improve their competitiveness. In this context, the importance of research the relationship between supplier development and improve the competitive capacity through innovation is analyzed. The objective of this article is to realize a systematic review of the literature related to supplier development and competitiveness, particularly, innovation as a competitive advantage in industrial or manufacturing environments. The results were obtained from scientific articles retrieved from Ebsco, Scopus, Web of science and Google Scholar databases that were published between 2017 and 2021. A selection process was followed with the PRISMA methodology. The results showed the research trending was increasing in the last 5 years, mainly in developed countries. Mexico has a minimal participation in these investigations.

Keywords: supply chain management competitiveness, supplier development, innovation.

JEL: L14, 021, 031. 


\section{INTRODUCCIÓN}

En la actualidad las firmas del sector industrial han puesto énfasis en el desempeño de sus cadenas de suministro a nivel internacional. El análisis de la competencia pasó de estudiar a la empresa de manera individual a visualizar la cadena de suministro en su totalidad.

La administración ideal de la cadena de suministro debe considerar desde el diseño, planeación, ejecución, monitoreo y control de las actividades tanto propias como de los actores estratégicos con el propósito de crear valor, construir una infraestructura sólida en comparación con los competidores y evaluar su desempeño a nivel internacional (APICS, 2015). Por tal motivo, se ha incrementado la cantidad de empresas industriales que realizan alianzas estratégicas con sus proveedores para establecer y mantener ventajas competitivas.

Otra tendencia en los estudios de administración es la gestión del conocimiento. Previamente la atención se ha focalizado en el proceso o flujo de productos, mientras que hoy en día se centra en el flujo de información. En la literatura se observa una estrecha relación entre la gestión del conocimiento y la innovación, ambas se consideran como factores relevantes de ventaja competitiva (Chen et al., 2018).

Este artículo tiene el propósito de revisar los estudios sobre las alianzas estratégicas entre compradores y proveedores, principalmente la gestión y actividades de desarrollo que realizan las empresas compradoras para analizar su relación con la innovación y competitividad.

Para lograr esto, se realizará una revisión sistemática de la literatura (RSL), técnica que es utilizada para identificar, evaluar e interpretar la literatura científica disponible de relevancia para responder preguntas de investigación (Kitchenham, 2004).

Por lo tanto, la pregunta principal de la presente investigación es: ¿cuál es la evidencia existente de estudios del desarrollo de proveedores en entornos de manufactura y su incidencia con la innovación y competitividad?
El objetivo principal es identificar los estudios relevantes sobre la temática de desarrollo de proveedores y su incidencia con la innovación y competitividad de las empresas manufactureras.

El artículo se compone de cuatro módulos adicionales a la introducción. En la primera parte se expone el marco teórico en el cual se basa la investigación y los conceptos relevantes sobre el desarrollo de proveedores, la competitividad y la innovación; después se explica la metodología que se siguió para la revisión de la literatura y los resultados. Finalmente se presentan las conclusiones y sugerencias sobre las líneas futuras de investigación relacionadas con esta temática.

\section{MARCO TEÓRICO}

La teoría de Michael Porter ha sido punto de partida de diversos estudios de las ciencias administrativas debido a la propuesta de un conjunto de elementos en sectores específicos con miras a sostener una ventaja competitiva. Entre estos elementos destaca el análisis de las relaciones estratégicas y la integración entre empresas relacionadas a diferencia de los estudios previos basados en empresas e industrias aisladas (Porter, 2007).

Otra de las teorías que justifican la presente investigación es la teoría basada en recursos que examina los recursos internos de las empresas enfocándose en formular estrategias para alcanzar ventajas competitivas sostenibles. De acuerdo con esta teoría estos recursos o capacidades deben aportar valor, ser raros, de imperfecta imitación y no sustituibles. Los recursos deben optimizar la eficiencia y efectividad de la organización, mejorar la satisfacción del cliente o reducir costos en relación con la competencia, en otras palabras, para que el recurso sea de valor debe mejorar el desempeño de la organización en relación con sus competidores (Barney, 1991; Madhani, 2010).

De manera similar, la teoría de capacidades dinámicas estudia cómo los recursos y capacidades deben cambiar o actualizarse a través del tiempo para mantener su relevancia en el mercado cambiante, mediante la creación de nuevos recursos, 
renovación o alteración de su mezcla de recursos. Dentro de esta teoría se identifican cuatro tipos de capacidades: capacidad de aprendizaje, capacidad de adaptación, capacidad de absorción y capacidad de innovación (Teece et al., 1997; Wang y Ahmed, 2007).

La teoría de las capacidades dinámicas está conectada con la teoría de la gestión del conocimiento, la cual proporciona una descripción de las actividades específicas que relacionan la administración del conocimiento con la competitividad a través del aprendizaje y la innovación (Chen et al., 2018). Para explicar cómo las actividades de la gestión del conocimiento resultan en una mayor competitividad Holsapple y Singh (2001) desarrollaron la teoría de la cadena de conocimiento, la cual explica cómo las actividades de gestión del conocimiento que se realizan en eventos de gestión del conocimiento resultan en una mayor competitividad empresarial.

Chen et al. (2015) realizaron un estudio partiendo de la teoría de la cadena de conocimiento y el desarrollo de proveedores para comprender la naturaleza de las actividades de gestión del conocimiento de compradores y proveedores que están integradas en las prácticas de desarrollo de proveedores, donde se explica por qué las actividades de gestión de conocimiento de clases distintas permiten a una empresa aprovechar su conocimiento para obtener una ventaja competitiva.

\subsection{Desarrollo de proveedores}

La necesidad de crear nuevas fuentes de proveeduría estratégica no es un tema reciente, Heinritz (1959) estableció las condiciones en que la responsabilidad del comprador pasaría de seleccionador de fuentes de abastecimiento a su creador, principalmente cuando la operación requiriera reducir distancias entre el proveedor y la planta, cuando los precios estuvieran fuera del presupuesto, cuando se necesitara incrementar capacidades de producción, o cuando los proveedores potenciales simplemente no estuvieran interesados en negocios adicionales.
Leenders (1966) nombró a este supuesto desarrollo de proveedores y proporcionó una visión más amplia definiéndolo como una situación en la que el comprador puede ver beneficios inusuales para el proveedor potencial que el mismo proveedor desconoce, tales como asistencia tecnológica, financiera y administrativa del comprador, incluyendo la posibilidad de negocios futuros como resultado de las habilidades adquiridas en este trabajo.

Krause et al. (1998) es uno de los referentes de investigaciones sobre desarrollo de proveedores en entornos industriales, lo define como el conjunto de actividades que realiza la empresa compradora con el fin de identificar, medir y mejorar el desempeño del proveedor y facilitar la mejora continua del valor de los bienes y servicios suministrados a la empresa compradora. Sunil y Routroy (2018) lo perciben como una iniciativa de la empresa compradora cuya función consiste en asistir a los proveedores clave a contribuir de manera competitiva de conformidad con los requisitos de los clientes.

Chen et al. (2018) especifican que son las actividades relacionadas con la gestión del conocimiento que desarrollan las empresas compradoras y proveedoras con el propósito de satisfacer las necesidades de suministro de la empresa compradora mejorando también el desempeño de la empresa proveedora. Sulungbudi et al. (2019) también identifican la gestión del conocimiento como un elemento de valor que permite desarrollar, coordinar e integrar una serie de capacidades que podría conducir a mejoras de desempeño, tanto de la empresa compradora como de los proveedores.

Como se observa en las definiciones previas, lo que ha cambiado es la inclusión de elementos en el desarrollo de proveedores, uno de los principales es la gestión del conocimiento que se adquiere a través de las relaciones estratégicas, lo que se considera de valor para el desarrollo de nuevas capacidades.

Acorde a diferentes autores (Aura y Juma, 2020; Calignano y Vaaland, 2017; Dalvi y Kant, 2018; O'Connor et al., 2018; Sulungbudi et al., 2019; Van der Westhuizen 
y Ntshingila, 2020) las diferentes actividades de desarrollo de proveedores abarcan la adquisición, selección, generación, asimilación y emisión de conocimientos, así como actividades de medición de conocimiento, liderazgo, coordinación y control.

Durante procesos de participación directa, tanto el proveedor como la empresa compradora comparten y sustituyen sus conocimientos cuando, desde su perspectiva, estos les aportan valor (Lee et al., 2018). Este comportamiento cumple con la teoría basada en recursos, la teoría de capacidades dinámicas y la teoría de la gestión del conocimiento.

El intercambio de información dentro del desarrollo de proveedores es un proceso que les permite tanto a la empresa compradora como a sus proveedores crear nuevos conocimientos (Lee et al., 2018; Wiratmadja y Tahir, 2021). Cabe mencionar, que la innovación está estrechamente relacionada con el concepto de creación de conocimiento, que a su vez se relaciona con la mejora del desempeño y de la competitividad de las organizaciones.

Una implementación exitosa de los programas de desarrollo de proveedores depende en gran medida de la calidad del conocimiento adquirido a través del intercambio de conocimiento. La aportación de nuevas ideas, enfoques y culturas favorece la innovación empresarial (Darroch y McNaughton, 2002). De esta manera, la eficacia de las actividades de intercambio de conocimientos entre la empresa y su proveedor contribuye a la competitividad de la cadena de suministro.

\subsection{Competitividad y Desarrollo de Proveedores}

En estos tiempos de evolución tecnológica acelerada las empresas deben renovarse y adaptarse a los cambios para su supervivencia. Además de ofrecer nuevos productos y servicios, es indispensable ajustar un cambio en la gestión empresarial que permita adaptarse con mayor facilidad. Esto se puede alcanzar mediante la adaptación de procesos y prácticas organizacionales que generen una ventaja competitiva (Teece et al., 1997; Vaccaro et al., 2012; Zhang et al., 2019).

Como se ha venido mencionando, los programas de desarrollo de proveedores son programas de relaciones estratégicas, la empresa compradora espera obtener una ventaja competitiva al transformar sus activos generales en recursos y capacidades específicas. Las transacciones entre comprador y proveedor se convierten en el recurso único del comprador, permitiéndole lograr y mantener una ventaja competitiva. Además, el proveedor se involucra en el proceso de desarrollo de productos y procesos de la empresa compradora, estrechando sus relaciones y fortaleciendo su colaboración (Manzoor et al., 2019).

La teoría basada en recursos expone que una empresa con recursos y capacidades únicas puede obtener una posición competitiva sostenible y un desempeño superior en un mercado determinado; a partir de allí, se deduce entonces que una empresa con capacidades de innovación podría lograr su objetivo de posicionarse de manera sostenible y superar a sus competidores en un mercado cambiante (Barney, 1991; Zhang et al., 2019).

Acorde a Teece et al. (1997), lo que determina el éxito de una empresa en mercados cambiantes, están representados por los recursos que pueda reunir y su habilidad para encontrar o crear competencias distintivas. Estos autores, abordan los tipos de procesos organizacionales, de los cuales es posible identificar tres tipos: procesos de integración, que engloba las alianzas estratégicas, la relación comprador-proveedor y el abastecimiento estratégico; procesos de aprendizaje, asociados a las habilidades organizacionales, a los nuevos procedimientos y una nueva lógica organizacional; los procesos de transformación que plantean la necesidad de reestructurar los recursos de la empresa mediante una transformación tanto interna como externa minimizando los costos de transacción.

Por otra parte, Wang y Ahmed (2007) al estudiar las capacidades dinámicas, señalan que el desempeño de las organizaciones empresariales se da a partir de la capacidad que tienen para alcanzar y sostener ventajas 
competitivas; o bien, su habilidad para identificar y capitalizar oportunidades. Dentro de las capacidades que analizan se encuentra la capacidad de innovación.

\subsection{Innovación}

Diversos estudios han tratado de definir la capacidad de innovación en relación con la gestión empresarial (Crossan y Apaydin, 2010; Lawson y Samson, 2001; Mol y Birkinshaw, 2009; M. Zhang y Hartley, 2018).

La definición que mejor se adapta al tema de investigación es la de Lawson y Samson (2001), quienes la definen como la capacidad de transformar continuamente el conocimiento y las ideas en nuevos productos, procesos y sistemas para el beneficio de la empresa y sus grupos de interés, donde se encuentran los proveedores. Para Zhang et al. (2019), la innovación representa la mejor opción de supervivencia a largo plazo de las organizaciones empresariales en mercados cambiantes.

Teece (2010) menciona un tipo de innovación en la que las organizaciones descubren y buscan oportunidades únicas, la cual define como innovación de modelos de negocio. En la literatura asociada a la administración, adquieren relevancia otros tipos de innovación adicionales entre los que se encuentra la innovación empresarial, de productos, tecnológica y de procesos (Yang et al., 2018).

Tanto la innovación empresarial como la innovación tecnológica son factores que inciden en el desempeño de las organizaciones. Hamel (2006) afirma que la innovación empresarial representa una de las fuentes más destacadas de ventaja competitiva y desempeño sostenible en el ámbito empresarial. Yang et al., (2018) argumentan que la innovación tecnológica es una herramienta necesaria para una posición sostenible en la era actual de globalización.

No siempre la innovación empresarial y tecnológica contribuyen directamente al desempeño de la empresa. En ese sentido, ambos tipos de innovación ayudan a las organizaciones a adquirir nuevos procesos que a su vez pueden resultar en un incremento en su rentabilidad y se podría argumentar que ambos tipos de innovación son complementarios. No obstante, los cambios no se relacionan directamente con la oferta de nuevos productos y servicios, sino también con la modificación del entorno de la gestión empresarial al interior de las organizaciones.

Por esa razón, también es relevante estudiar el impacto de la innovación a través de la investigación y desarrollo en el desempeño de las empresas, deduciendo que la innovación empresarial permite a las organizaciones adoptar procesos innovadores y tecnológicos necesarios para el buen funcionamiento de las actividades operativas (Nemlioglu y Mallick, 2017),.

Para Hinterhuber y Liozu (2014), un sistema de gestión innovador debe considerar diferentes políticas y procedimientos que permita utilizar los recursos de manera eficaz e impulsado a las organizaciones a ganar posiciones competitivas sostenibles. Estos autores, analizan el término de innovación en los precios, proporcionándole una relevancia significativa similar a la innovación de productos o la innovación de modelos de negocio.

La innovación empresarial asiste a la organización a alcanzar altos rendimientos y a mejorar su desempeño a través de la integración de prácticas novedosas (Mol \& Birkinshaw, 2009; Yang et al., 2018).

La innovación empresarial y la innovación de modelos de negocio no forman parte del mismo concepto. La innovación de modelos de negocio se define como la necesidad de transformar significativamente el modelo de negocio existente, en otras palabras, la propuesta de valor de la empresa. En este sentido, se considera a las redes de valor, la creación de valor y la captura de valor como componentes primarios del modelo de negocio (Koen et al., 2011).

Un modelo de negocio permite lograr eficiencia, desarrollar objetivos estratégicos y valor para todas las partes interesadas Partiendo de esta afirmación, Matt et al. (2015) explican que el elemento de oferta de valor captura la propuesta de valor de la empresa, así como su posicionamiento competitivo en el mercado. La creación de valor se refiere a cómo la organización se dará 
cuenta de la oferta de valor al identificar las competencias y los recursos centrales de la organización. Asimismo, la creación de valor se centra en la estructura organizativa, los canales de distribución, las actividades internas y la cadena de valor. Este último concepto se relaciona directamente con la administración de la cadena de suministro.

Se concluye que una organización debe ser innovadora en términos de mejora del desarrollo de nuevos productos, servicios o procesos, lo que se conoce como innovación empresarial. Además, es necesario que desarrolle nuevos conocimientos, habilidades y competencias de manera constante. Por último, es necesario tener presente que las alianzas estratégicas, como el desarrollo de proveedores, tienen un papel relevante en todo lo anterior; por ello, es importante que se mejoren las capacidades, y se de un enfoque hacia la innovación continua, con miras a ser capaces de responder rápidamente a los cambios en el mercado (Teece et al., 1997; Van Tonder et al., 2020).
Como primer paso de la metodología se identifican las variables de estudio. Como variable independiente, el desarrollo de proveedores; variable dependiente, la competitividad y como variable mediadora, la innovación.

El alcance de la investigación es un estudio exploratorio, no por ser un tema poco estudiado, mas bien con el propósito de abordar el tema del desarrollo de proveedores desde una perspectiva diferente a la que se ha estudiado hasta ahora e identificar variables prometedoras para futuras investigaciones. También se considera de alcance descriptivo por captar el contexto actual, recoge información sobre las teorías, conceptos de desarrollo de proveedores, competitividad e innovación y brinda datos para determinar su operacionalización quedando como precedente para estudios correlacionales.

El diseño de la investigación es no experimental longitudinal, ya que se desea observar la evolución de la literatura científica de las variables a lo largo de los últimos años.

Figura 1. Diagrama de flujo PRISMA

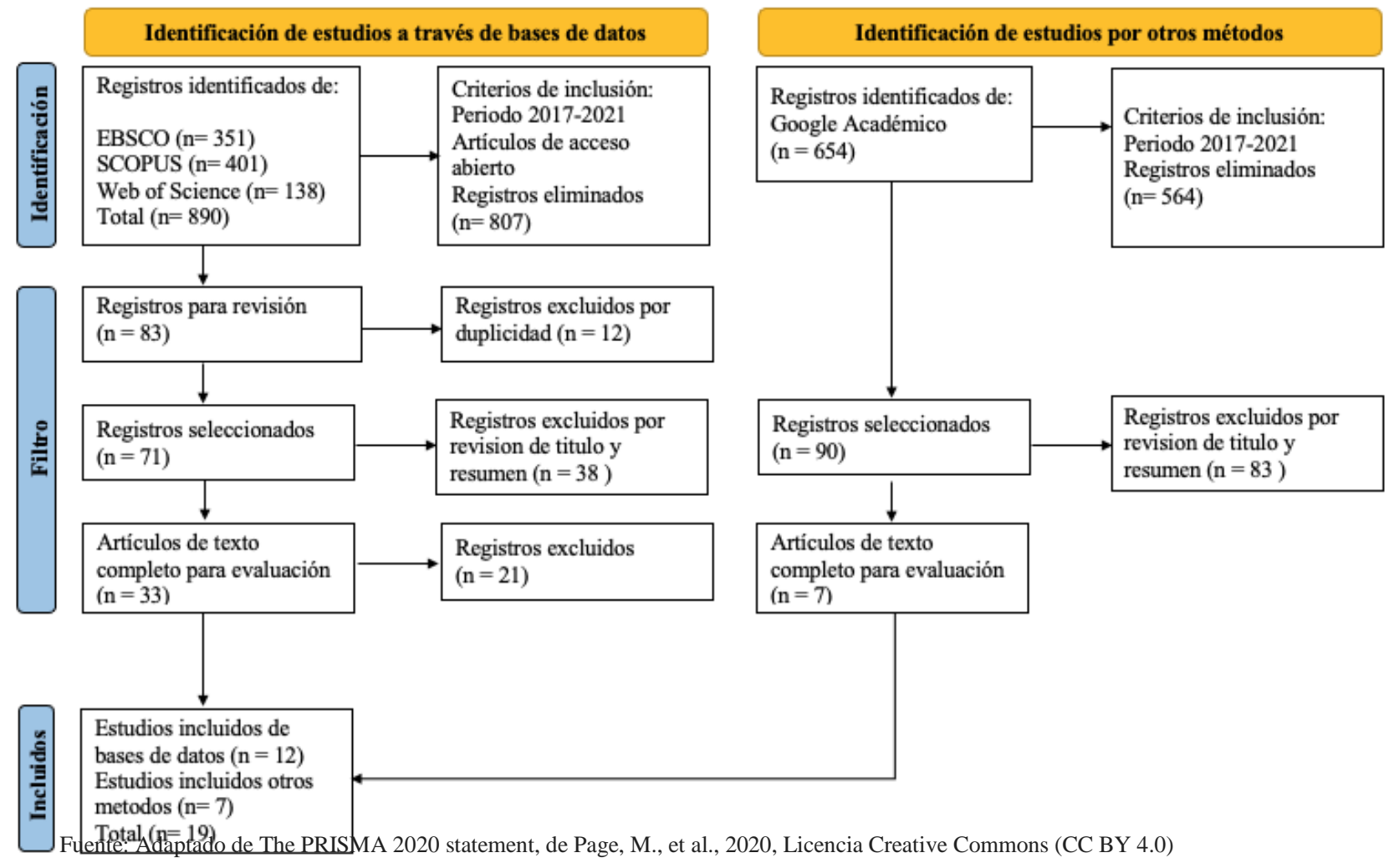


puesto que la selección de los artículos dependerá de que cumplan con los criterios previamente establecidos para el desarrollo de la investigación. Por esta razón se consideró una revisión sistemática de la literatura siguiendo los procedimientos de Kitchenham (2004) en tres etapas, identificación, filtro e inclusión mediante el diagrama de flujo PRISMA como se observa en la figura 1 (Page et al., 2021; PRISMA, 2020).

En la etapa de identificación, se diseñó la estrategia de búsqueda de información de la revisión de literatura científica a partir de la pregunta de investigación, para ello se seleccionaron las bases de datos científicas: Ebsco, Scopus, Web of science y Google Académico.

Posteriormente, se definieron los criterios de búsqueda bajo los términos equivalentes a desarrollo de proveedores (supplier development, vendor development, supplier management).

En el caso del criterio de manufactura debido a que en la literatura también se utilizan términos equivalentes como manufacture o manufacturing, se utilizó manufactur*. En los casos de los criterios de competitividad e innovación, se utilizaron sus vocablos en el idioma inglés (competitiveness, innovation).

En la base de datos Web of science, se obtuvo la siguiente cadena de búsqueda $T I$ $=($ "supplier development") $O R \quad T I=(" v e n d o r$ development") OR TI=("supplier management") AND TS=(competitiveness AND manufactur* AND innovation), obteniendo 138 resultados.

Para EBSCO se utilizó como cadena de búsqueda TI "supplier development" OR TI "vendor development" OR TI "supplier management" AND TX competitiveness AND TX manufactur* AND TX innovation, se obtuvo 351 resultados.

En la base de datos de SCOPUS, la cadena de búsqueda se determinó de la siguiente manera: title "supplier development" OR title "vendor development" OR title "supplier management" AND competitiveness AND innovation AND manufactur*. Con esta búsqueda se obtuvieron 401 resultados.

El paso siguiente consistió en el filtro a partir de limitadores de las bases de datos, tales como limitadores temporales (20172021), publicaciones arbitradas y en los casos en que se habilitó la opción, artículos de libre acceso.

Con estos ajustes, se identificaron 44 resultados en Ebsco, 21 resultados en Scopus y 18 resultados en Web of science. Se utilizó la herramienta Rayyan para la gestión de los criterios de inclusión y exclusión. Una vez que se obtuvieron los resultados de las bases de datos, se exportaron a archivos compatibles para su revisión en la herramienta de gestión Rayyan. En este paso se exportaron 83 resultados.

La búsqueda en Google Académico se realizó de manera híbrida. La primera parte fue automática con la siguiente cadena de búsqueda: "supplier development" $O R$ "vendor development" OR "supplier management" + "competitiveness" + "manufactur*" + innovation. Como primera respuesta se obtuvieron 654 resultados, posteriormente se ajustó el periodo de tiempo y se agregó el operador booleano - "green" para evitar estudios que abordan prácticas de sustentabilidad que no son parte del enfoque de este artículo. Con ello, se obtuvieron 90 resultados. Después se realizó un filtro manual para seleccionar sólo los artículos y capítulos de libros de acceso abierto. Se observó que aun cuando se seleccionó el operador booleano de - "green" se incluyeron algunos resultados con esta temática, los cuales fueron eliminados manualmente en este paso. Durante esta revisión se estuvieron revisando resúmenes y al final se seleccionaron 7 resultados para la siguiente fase, los cuales se exportaron al gestor Rayyan.

En el gestor Rayyan se seleccionó la opción de detectar duplicados. Del total de los 90 resultados (83 resultados de Ebsco, Scopus, Web of science y 7 resultados de Google Académico) se identificaron 12 resultados duplicados, los cuales fueron removidos, quedando 71 resultados para el siguiente filtro que consistió en leer todos los títulos y resúmenes para identificar si responden a las siguientes preguntas: ¿el estudio aborda el desarrollo de proveedores o relaciones estratégicas con proveedores?, ¿la 
investigación menciona la variable de competitividad, innovación o ambas?, ¿la investigación se realizó en entornos industriales?

Al concluir este filtro, se seleccionaron 40 artículos para la revisión de texto completo, de los cuales se excluyeron 21 resultados, debido a que el estudio no se enfocó en el sector de manufactura, o bien no mencionó la relación del desarrollo de proveedores con la innovación o la competitividad.

\section{RESULTADOS}

\subsection{Análisis Bibliométrico}

Se elaboró un análisis bibliométrico con algunas funciones de las bases de datos que permitieron analizar gráficamente sus resultados.

Tal es el caso de Scopus, que permite observar con relación a los 401 artículos recuperados como su tasa de crecimiento ha ido en aumento desde el año 2000, año en el que se identifican las primeras publicaciones en esta base de datos. El crecimiento del 2000 al 2011 fue paulatinamente, en el 2012 superó las 10 publicaciones anuales, en 2016 superó las 20 publicaciones, y a partir del 2017 el crecimiento fue exponencial. científicos con esta temática, casi el $25 \%$ del total de las publicaciones durante los últimos 20 años. El presente artículo se realizó en los primeros meses de 2021, lo que justifica que la cantidad de artículos con respecto al año anterior fuera menor.

En Web of science también se analizó el comportamiento de las publicaciones por año a través de la herramienta Bibliometrix. De los 138 artículos, el periodo fue desde 1996 a 2021. Previo a 2007 las publicaciones fueron menores a 5 artículos anuales, a partir de 2007 a 2014 se mantienen en alrededor de 5 publicaciones anuales, y en 2015 se observa un incremento hasta llegar a las 10 publicaciones. Durante el 2018 fue el periodo de mayor producción alcanzando 19 artículos.

Para analizar el comportamiento de los artículos de la base de datos de Ebsco se tuvieron ciertas restricciones ya que la base de datos no es compatible con la herramienta Bibliometrix, en cambio se utilizó la herramienta Rayyan para este análisis. Se observaron dos picos de la literatura en dos diferentes periodos entre 2014 a 2021, el primero de ellos fue en el 2015 donde alcanzó 13 publicaciones anuales, luego se mantuvo en 12 publicaciones anuales y para el 2020 alcanza nuevamente 13 artículos anuales.

Figura 2. Publicaciones por año

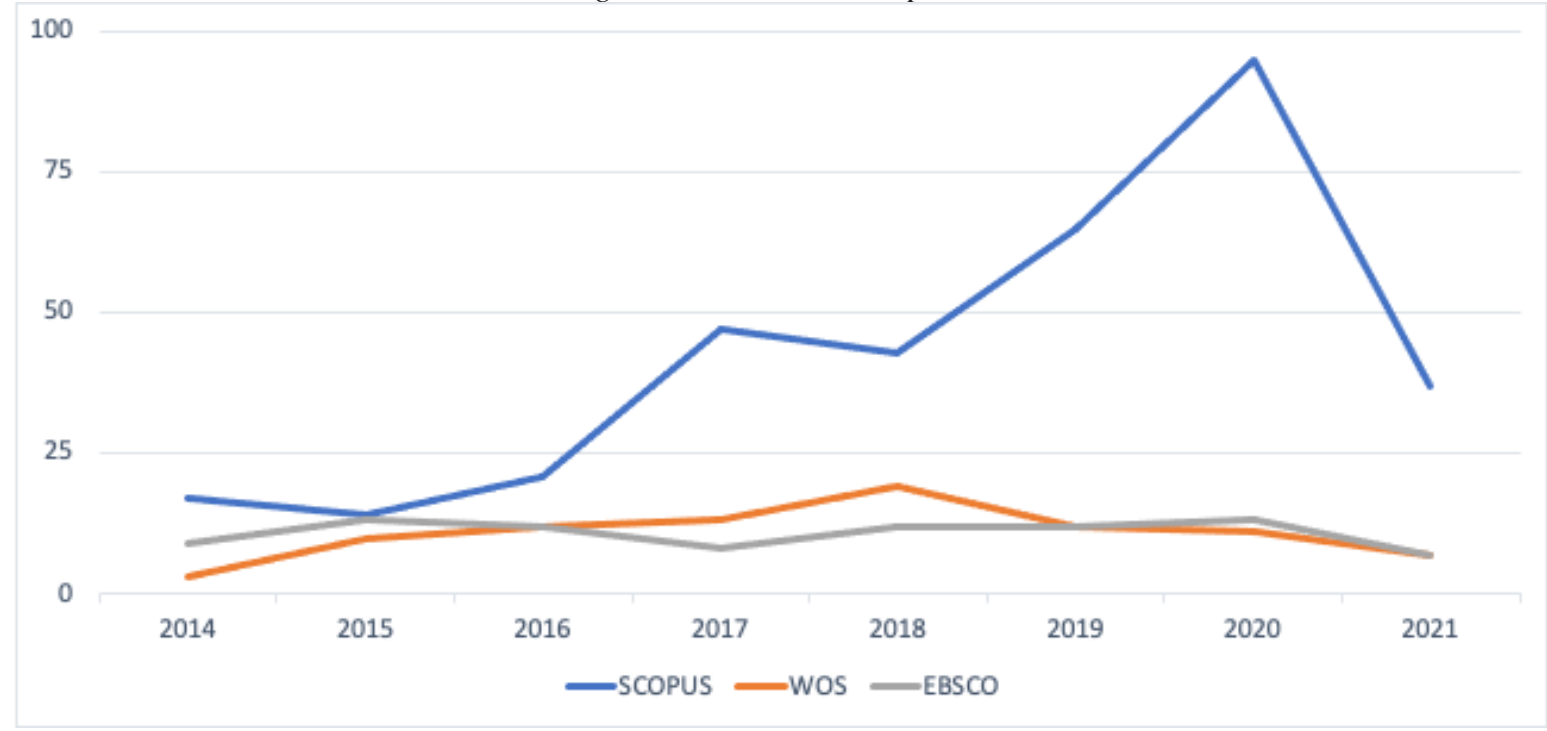

En el 2020, se publicaron 95 artículos

En la figura 2 se muestran las

Fuente: Elaboración propia 
tendencias de la literatura recuperada de las tres bases de datos, se observa que el comportamiento de los artículos revisados cumple con la ley sobre el crecimiento de la ciencia establecida por Derek Price.

El modelo de Price establece cuatro fases de crecimiento científico a lo largo del tiempo, la primera la determina como fase de desarrollo constante, la segunda fase es de crecimiento lineal, la tercera fase de crecimiento exponencial y la cuarta fase de estabilización (Price, 1963).

Dentro de este orden de ideas, la recopilación de Scopus se encuentra en la tercera fase de crecimiento científico y tanto los artículos de Web of science como los de Ebsco aún se encuentran en la fase de crecimiento constante.

Otro resultado relevante que se encontró en la base de datos de Scopus es la clasificación de artículos por área de estudio con la temática seleccionada, el mayor porcentaje de publicaciones fue con enfoque en áreas de administración y negocios con el $33 \%$, siguiendo en orden de importancia ciencias de la ingeniería con el $15 \%$, ciencias de decisión con 13\%, ciencias ambientales contó con $8 \%$ de participación al igual que las ciencias sociales con el $8 \%$.

También se realizó un análisis de las publicaciones científicas por países. En Scopus, se observó dentro de los primeros 10 países que el 50\% son países europeos (Reino Unido, Francia, Alemania, España e Italia), en segundo lugar, se encontraron países asiáticos (China e India) en la tercera y cuarta posición respectivamente. En Web of science, también el 50\% de los países en las primeras 10 posiciones son europeos (Alemania, Reino Unido, Irlanda, Suiza y España).

Coincidentemente se encuentran en segundo lugar los países asiáticos (China, India y Malasia) con 78 publicaciones, sin embargo, los países de la región de Norteamérica (Estados Unidos y Canadá) tienen una posición considerable con 77 publicaciones en conjunto.

Estados Unidos sobresale en ambas bases de datos, en Scopus obteniendo el segundo lugar con 111 publicaciones y Web of science el primer lugar con 59 publicaciones.
México, se encuentra en la posición 38 con 3 publicaciones en Scopus y la 29 en Web of science con 1 publicación. Los resultados se pueden observar en las tablas 1 y 2 .

$\underline{\text { Tabla 1. Publicaciones por países en Scopus }}$

\begin{tabular}{rlr}
\hline \multicolumn{2}{l}{ Scopus } & \\
\hline & País & Publicaciones \\
\hline 1 & Reino Unido & 137 \\
2 & Estados Unidos & 111 \\
3 & China & 108 \\
4 & India & 105 \\
5 & Francia & 26 \\
6 & Alemania & 26 \\
7 & España & 26 \\
8 & Brasil & 24 \\
9 & Italia & 24 \\
10 & Iran & 23
\end{tabular}

Tabla 2. Publicaciones por países en WoS

\begin{tabular}{rrr}
\hline \multicolumn{2}{l}{ Web of science } & \\
\hline \multicolumn{2}{l}{ País } & Publicaciones \\
\hline 1 Estados Unidos & 59 \\
2 & China & 50 \\
3 & Alemania & 29 \\
4 & Reino Unido & 27 \\
5 & India & 21 \\
6 & Canada & 18 \\
7 & Irlanda & 10 \\
8 & Suiza & 9 \\
9 & España & 8 \\
10 & Malasia & 7
\end{tabular}

Fuente: Elaboración propia

Las tablas 3 y 4 muestran el listado de los autores con mayor impacto, acorde a su número de artículos publicados y el valor de sus índices $\mathrm{g} \mathrm{y} \mathrm{h}$, estos indicadores se han empleado para señalar la calidad de las producciones científicas de los autores. El índice $\mathrm{h}$, se basa en que a partir del número de citas que recibe el autor representa un mejor indicador de la calidad de su investigación, más allá de las cantidades de las publicaciones que realiza. Por otro lado, el índice g indica la calidad general del autor mediante el rendimiento de sus artículos principales, de cierta manera es una modificación del índice $\mathrm{h}$ 
(Egghe, 2006; Hirsch y Buela-Casal, 2014).

$\mathrm{El}$ autor con mayor impacto en Scopus fue Angappa Gunasekaran, quien se especializa en temas de cadena de suministro, su artículo más citado recuperado de Scopus es Information technology for competitive advantage within logistics and supply chains: A review publicado en el 2017. Al realizar el análisis con la base de datos de Web of science con Bibliometrix, también se encontró como el autor más relevante por cantidad de citas con 10 artículos, pero al revisar sus índices h y g no se encontró en las primeras posiciones. Se hizo la correlación con su relevancia en Google Académico, ya que esta plataforma proporciona los datos por autor, se encontró que ha sido citado 31,025 veces desde 2016 y su índice h es de 91, su artículo más citado es A framework for supply chain performance measurement publicado en 2004.

El autor más relevante en Web of science fue Stephan Wagner con enfoque en relaciones de proveedores. En Google Academico ha sido citado 14,080 veces, su índice h es 60, y su artículo más citado fue $A n$ empirical examination of supply chain performance along several dimensions of risk publicado en 2008.

La herramienta Bibliometrix también permitió identificar los artículos de desarrollo de proveedores, innovación y competitividad con mayor relevancia clasificados según sus citas a nivel global.

Tabla 3. Autores relevantes WoS

\begin{tabular}{lccc}
\hline \multicolumn{4}{c}{ Web of science } \\
\hline Autor & $\begin{array}{c}\text { Índice } \\
\mathrm{h}\end{array}$ & $\begin{array}{c}\text { Índice } \\
\mathrm{g}\end{array}$ & $\begin{array}{c}\text { No. } \\
\text { Art. }\end{array}$ \\
\hline Wagner, S. & 7 & 7 & 7 \\
Sarkis, J. & 6 & 6 & 6 \\
Bai, C. & 4 & 5 & 5 \\
Kumar, C. & 3 & 4 & 4 \\
Li, W. & 4 & 4 & 4 \\
Routroy, S. & 3 & 4 & 4 \\
Chen, L. & 3 & 3 & 3 \\
Cheng, T. & 3 & 3 & 3 \\
Dalvi, M. & 3 & 3 & 3 \\
Glock, C. & 3 & 3 & 3
\end{tabular}

Tabla 4. Autores relevantes Scopus

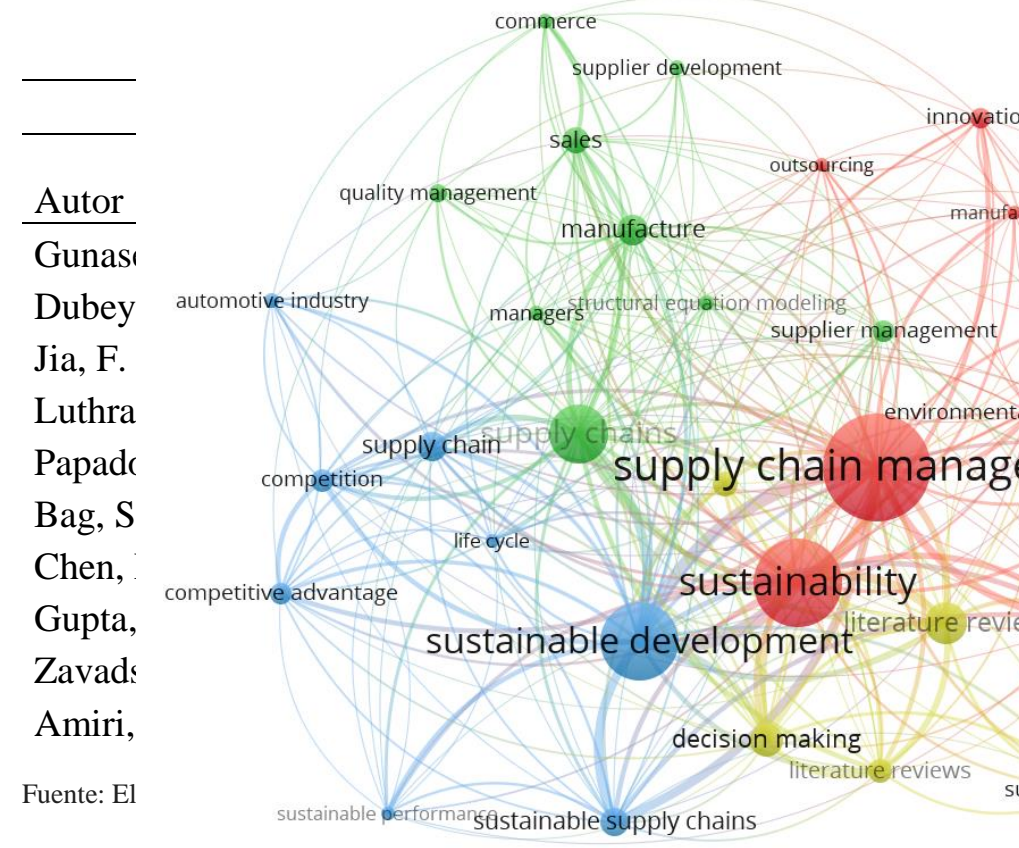

El articulo con mayor relevancia tue

The relationships between supplier development, commitment, social capital accumulation and performance improvement (D. R. Krause et al., 2007) citado 649 veces en Scopus, 667 veces en Web of science y 1401 veces en Google Académico, el cual investiga la relación entre el desarrollo de proveedores de las empresas compradoras estadounidenses, el compromiso, la acumulación de capital social con proveedores clave y el desempeño de las empresas compradoras. Respalda la teoría de que el compromiso de las empresas compradoras con proveedores clave pueden mejorar el desempeño de la empresa compradora.

El otro artículo que sobresalió fue The dark side of buyer-supplier relationships: A social capital perspective (Villena et al., 2011) citado 471 veces en Scopus y 856 en Google Académico, el cual expone cómo la literatura sobre la administración de la cadena de suministro suele promover el lado positivo de las relaciones entre comprador y proveedor, si bien confirma que las relaciones colaborativas afectan positivamente el desempeño del comprador, cuando se lleva al extremo puede reducir la capacidad del comprador para ser objetivo y tomar decisiones efectivas, así como aumentar el comportamiento oportunista del proveedor. 
Otro Fuente: Elaboración propia coocurrencia de palabras claves, el cual permite identificar el desarrollo de temas de investigación y puntos de interés en campos específicos (Goksu, 2021). Se utilizó la herramienta VOS viewer y el resultado se observa en la figura 3 . Se aprecia que las investigaciones se han concentrado en temas de administración de la cadena de suministro (supply chain management) y también abordan temáticas de desarrollo sustentable (sustainable development, sustainability). A menor escala se observan los temas seleccionados, desarrollo de proveedores, innovación, manufactura y competitividad.

En términos generales del total de artículos recuperados se pudo obtener la evolución temática a través de los últimos 20 años como se observa en la figura 4.
Del 2000 al 2010, las investigaciones que abordaban el desarrollo de proveedores, competitividad e innovación se focalizan en temas como satisfacción de proveedores, gerencia y personal. A partir del 2011 se ve la transición hacia las investigaciones de desarrollos sostenible y cadenas de suministro.

\subsection{Revisión sistemática de la literatura}

El resultado de la RSL fue de 19 artículos para su análisis completo, en el total de la muestra se mencionó a la competitividad textualmente o con términos equivalentes como ventaja competitiva o competencia empresarial. En el caso del desarrollo de proveedores, fueron 16 artículos donde se abordó directamente este tema, algunos documentaron las relaciones estratégicas con los proveedores, o bien, la importancia de los proveedores en la cadena de suministro.

Figura 4. Evolución temática de la literatura 2000-2021

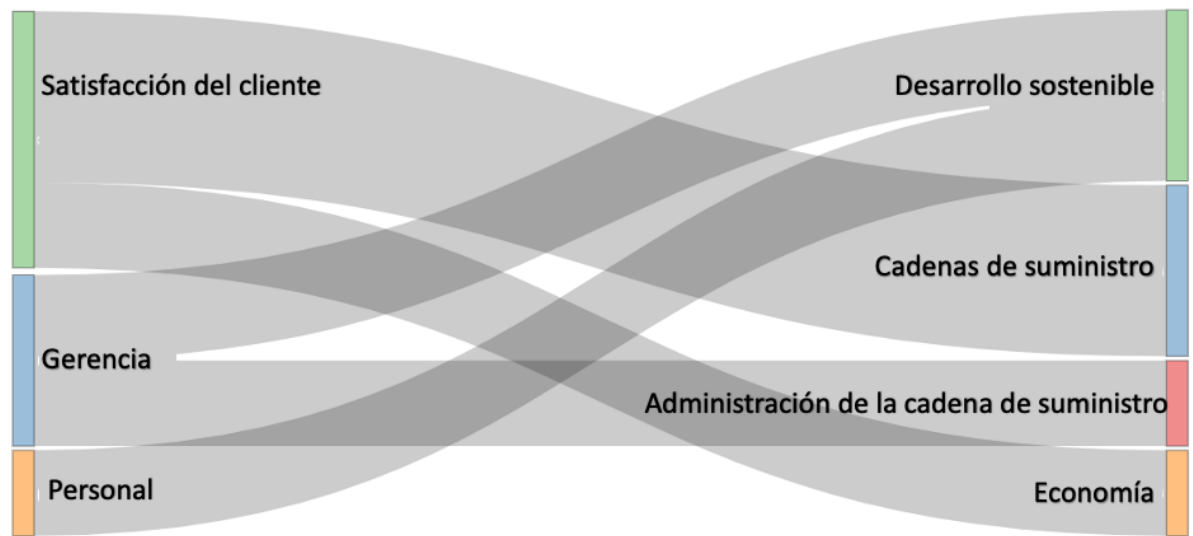

artículos de 
innovación empresarial, tecnológica, de productos e innovación en fijación de precios. En relación con la innovación en fijación de precios, se mencionó que ha sido subvalorada y poco explorada a pesar de ser una de las fuentes más eficaces de una empresa en términos de ventaja competitiva.

En términos generales, los artículos seleccionados abordan antecedentes y conceptos relevantes para la investigación, así como teorías que fueron consideradas durante la redacción de la primera parte del presente artículo.

En la tabla 5 se recopila la relación de los hallazgos de los artículos seleccionados en la RSL, indicando si abordan los conceptos de manera explícita, implícita o no se encontraron directamente en la literatura.
El estudio bibliométrico que se realizó proporcionó como resultado la identificación de áreas de oportunidad en la investigación de las ciencias administrativas con enfoque en cadena de suministro y proveeduría estratégica, en específico sobre el análisis de los estudios sobre cómo la innovación interfiere en la relación del desarrollo de proveedores y la competitividad.

La gestión del conocimiento es una variable recurrente cuando se estudian tópicos de innovación, y la teoría de la cadena de conocimiento proporciona un punto de partida para futuros análisis a desarrollar. También resulta relevante mencionar la poca exploración de la innovación de fijación de precios en estudios de competitividad, la cual presenta una alta relación con el desarrollo de proveedores y el desempeño de la empresa compradora.

Tabla 5. Estudios que se incluyeron en la revisión sistemática de literatura

\begin{tabular}{|c|c|c|c|}
\hline Autores & $\begin{array}{l}\text { Desarrollo de } \\
\text { proveedores }\end{array}$ & Competitividad & Innovación \\
\hline Amoako-Gyampah et al. (2019) & 1 & E & $E$ \\
\hline Arrfou (2019) & 1 & E & $\mathrm{E}$ \\
\hline Benton et al., (2020) & E & E & $\mathrm{N}$ \\
\hline Chen et al., (2018) & E & E & I \\
\hline Dastyar, Rippel, Pannek, et al. (2020) & E & I & $N$ \\
\hline Dastyar, Rippel, y Freitag (2020) & E & 1 & $\mathrm{~N}$ \\
\hline Glock et al. (2017) & E & 1 & $N$ \\
\hline Golmohammadi y Hassini (2021) & E & I & $N$ \\
\hline Islami et al. (2020) & I & E & $E$ \\
\hline Jain et al. (2020) & E & E & I \\
\hline Jin et al. (2019) & E & E & $N$ \\
\hline Lee et al. (2018) & E & E & I \\
\hline Lorentz et al. (2018) & E & 1 & $E$ \\
\hline Manzoor et al. (2019) & E & E & $N$ \\
\hline Proch et al. (2017) & E & I & E \\
\hline Saghiri y Wilding (2021) & E & E & $E$ \\
\hline Sunil y Routroy (2018) & E & 1 & 1 \\
\hline Wiratmadja y Tahir (2021) & E & E & $\mathrm{E}$ \\
\hline Zhang et al. (2017) & $\mathrm{E}$ & $\mathrm{E}$ & E \\
\hline Total & 16 & 19 & 10 \\
\hline
\end{tabular}

\section{CONCLUSIONES}

Fuente: Elaboración propia
Los estudios más recientes sobre 
desarrollo de proveedores coinciden en que se está depositando una mayor confianza en los proveedores para involucrarse en el diseño y desarrollo de nuevos productos, pero es necesario analizar cómo se pudiera agilizar el proceso de adopción de nuevas tecnologías por parte de los proveedores y la participación que la empresa compradora pueda tener.
Estas investigaciones se realizaron en los países desarrollados, así como en los países que se caracterizan por la industria manufacturera de exportación, como China, India, Malasia, Irán y Brasil.

México es un país que también se caracteriza por este tipo de industria, pero no ha tenido una participación relevante en estos estudios. Con base en los resultados, se deja un antecedente para futuras investigaciones en esta temática en el país.

\section{REFERENCIAS}

APICS. (2015, March 11). The Total Scope of Supply Chain Management. Association for Supply Chain Management. http://www.apics.org/sites/apics-blog/thinking-supply-chain-topic-searchresult/thinking-supply-chain/2015/03/11/the-total-scope-of-supply-chain-management

Aura, M., \& Juma, D. (2020). Influence of supplier development on operational performance of manufacturing firms in the Nairobi Securities Echange, Kenia. International Journal of Recent Research in Social Sciences and Humanities, 7(3), 88-105. https://www.paperpublications.org/upload/book/paperpdf-1599368891.pdf

Barney, J. (1991). Firm Resources and Sustained Competitive Advantage. Journal of Management, 17(1), 99-120. https://doi.org/10.1177/014920639101700108

Calignano, G., \& Vaaland, T. I. (2017). Supplier Development in Tanzania; Experiences, expectations and motivation. The Extractive Industries and Society, 4(2), 385-397. https://doi.org/10.1016/j.exis.2017.01.012

Chen, L., Ellis, S., \& Holsapple, C. (2015). Supplier Development: A Knowledge Management Perspective. In Knowledge and Process Management (Vol. 22, Issue 4, pp. 250-269). John Wiley and Sons Ltd. https://doi.org/10.1002/kpm.1478

Chen, L., Ellis, S., \& Holsapple, C. (2018). A knowledge management perspective of supplier development: Evidence from supply chain scholars and consultants. Knowledge \& Process Management, 25(4), 247-257. http://10.0.3.234/kpm.1566

Crossan, M. M., \& Apaydin, M. (2010). A Multi-Dimensional Framework of Organizational Innovation: A Systematic Review of the Literature. Journal of Management Studies, 47(6), 1154-1191. https://doi.org/10.1111/j.1467-6486.2009.00880.x

Dalvi, M. V., \& Kant, R. (2018). Effect of supplier development activities on performance outcomes: an empirical study. Benchmarking, 25(2), 489-516. https://doi.org/10.1108/BIJ-072016-0107

Darroch, J., \& McNaughton, R. (2002). Examining the link between knowledge management practices and types of innovation. Journal of Intellectual Capital, 3(3), 210-222. https://doi.org/10.1108/14691930210435570

Egghe, L. (2006). An improvement of the h-index: The g-index. ISSI Newsletter, 1-4. http://pds4.egloos.com/pds/200703/08/11/g_index.pdf

Goksu, I. (2021). Bibliometric mapping of mobile learning. Telematics and Informatics, 56, 101491. https://doi.org/10.1016/j.tele.2020.101491

Gunasekaran, A., Patel, C., \& McGaughey, R. E. (2004). A framework for supply chain performance measurement. International Journal of Production Economics, 87(3), 333-347. https://doi.org/10.1016/j.ijpe.2003.08.003

Gunasekaran, Angappa, Subramanian, N., \& Papadopoulos, T. (2017). Information technology for competitive advantage within logistics and supply chains: A review. Transportation Research Part E: Logistics and Transportation Review, 99, 14-33. https://doi.org/10.1016/j.tre.2016.12.008 
Hamel, G. (2006). The Why, What, and How of Management Innovation. Harvard Business Review, February, 1-15. http://he-product-images.s3.amazonaws.com/docs/R0602Cf2.pdf

Heinritz, S. (1959). Purchasing: Principles and applications (Third Edit). Prentice Hall.

Hinterhuber, A., \& Liozu, S. M. (2014). Is innovation in pricing your next source of competitive advantage? Business Horizons, 57(3), 413-423. https://doi.org/10.1016/j.bushor.2014.01.002

Hirsch, J. E., \& Buela-Casal, G. (2014). The meaning of the h-index. International Journal of Clinical and Health Psychology, 14(2), 161-164. https://doi.org/10.1016/S16972600(14)70050-X

Holsapple, C. W., \& Singh, M. (2001). Knowledge chain model: Activities for competitiveness. Expert Systems with Applications, 20(1), 77-98. https://doi.org/10.1016/S09574174(00)00050-6

Kitchenham, B. (2004). Procedures for Performing Systematic Reviews. In Procedures for Performing Systematic Reviews. https://doi.org/10.1145/3328905.3332505

Koen, P. A., Bertels, H. M. J., \& Elsum, I. R. (2011). The three faces of Business model innovation: Challenges for established firms. Research Technology Management, 54(3), 52-59. https://doi.org/10.5437/08953608X5403009

Krause, D., Handfield, R., \& Scannell, T. (1998). An empirical investigation of supplier development: Reactive and strategic processes. Journal of Operations Management, 17(1), 39-58. https://doi.org/10.1016/S0272-6963(98)00030-8

Krause, D. R., Handfield, R. B., \& Tyler, B. B. (2007). The relationships between supplier development, commitment, social capital accumulation and performance improvement. Journal of Operations Management, 25(2), 528-545. https://doi.org/10.1016/j.jom.2006.05.007

Lawson, B., \& Samson, D. (2001). Developing innovation capability in organisations: A dynamic capabilities approach. International Journal of Innovation Management, 05(03), 377-400. https://doi.org/10.1142/s1363919601000427

Leenders, M. (1966). Supplier Development. Journal of Purchasing, 2(4), 47-62. https://doi.org/10.1111/j.1745-493X.1966.tb00039.x

Madhani, P. (2010). Resource Based View (RBV) of Competitive Advantage: An Overview. In Resource Based View: Concepts and practices. http://papers.ssrn.com/sol3/papers.cfm?abstract_id=1578704

Matt, C., Hess, T., \& Benlian, A. (2015). Digital Transformation Strategies. Business \& Information Systems Engineering, 57(5), 339-343. https://doi.org/10.1007/s12599-015-0401-5

Mol, M. J., \& Birkinshaw, J. (2009). The sources of management innovation: When firms introduce new management practices. Journal of Business Research, 62(12), 1269-1280. https://doi.org/10.1016/j.jbusres.2009.01.001

Nemlioglu, I., \& Mallick, S. K. (2017). Do Managerial Practices Matter in Innovation and Firm Performance Relations? New Evidence from the UK. European Financial Management, 23(5), 1016-1061. https://doi.org/10.1111/eufm.12123

O’Connor, N. G., Yang, Z., \& Jiang, L. (2018). Challenges in gaining supply chain competitiveness: Supplier response strategies and determinants. Industrial Marketing Management, 72, 138-151. https://doi.org/10.1016/j.indmarman.2018.04.003

Page, M. J., McKenzie, J. E., Bossuyt, P. M., Boutron, I., Hoffmann, T. C., Mulrow, C. D., Shamseer, L., Tetzlaff, J. M., Akl, E. A., Brennan, S. E., Chou, R., Glanville, J., Grimshaw, J. M., Hróbjartsson, A., Lalu, M. M., Li, T., Loder, E. W., Mayo-Wilson, E., McDonald, S., ... Moher, D. (2021). The PRISMA 2020 statement: an updated guideline for reporting systematic reviews. BMJ, 372, n71. https://doi.org/10.1136/bmj.n71

Porter, M. E. (2007). La ventaja competitiva de las naciones. Harvard Business Review América Latina, Reimpresió, 4-23. https://es.calameo.com/read/00472423715ecda3a215b

Price, D. (1963). Little Science, Big Science. In Little Science, Big Science. Columbia University Press. https://doi.org/10.7312/pric91844 
PRISMA. (2020). Preferred Reporting Items for Systematic Reviews and Meta-Analyses. PRISMA 2020 Statement. http://prisma-statement.org/

Sulungbudi, M., Yanamandram, V., Akter, S., \& Tam, L. (2019). Supplier development: Practices and measurement. In A. G. Abdullah, I. Widiaty, \& C. U. Abdullah (Eds.), Global Competitiveness: Business Transformation in the Digital Era. Routledge. https://doi.org/10.1201/9780429202629

Teece, D. (2010). Business Models, Business Strategy and Innovation. Long Range Planning, 43(23), 172-194. https://doi.org/10.1016/j.lrp.2009.07.003

Teece, D., Pisano, G., \& Shuen, A. (1997). Dynamic capabilities and strategic management. Strategic Management Journal, 18(7), 509-533. https://doi.org/10.1142/9789812796929_0004

Vaccaro, I. G., Jansen, J. J. P., Van Den Bosch, F. A. J., \& Volberda, H. W. (2012). Management Innovation and Leadership: The Moderating Role of Organizational Size. Journal of Management Studies, 49(1), 28-51. https://doi.org/10.1111/j.1467-6486.2010.00976.x

Van der Westhuizen, J., \& Ntshingila, L. (2020). The effect of supplier selection, supplier development and information sharing on SME's business performance in Sedibeng. International Journal of Economics and Finance Studies, 12(2), 153-167. https://doi.org/10.34109/ijefs.202012203

Villena, V. H., Revilla, E., \& Choi, T. Y. (2011). The dark side of buyer-supplier relationships: A social capital perspective. Journal of Operations Management, 29(6), 561-576. https://doi.org/10.1016/j.jom.2010.09.001

Wagner, S., \& Bode, C. (2008). An Empirical Examination of Supply Chain Performance Along Several Dimensions of Risk. Journal of Business Logistics, 29(1), 307-325. https://doi.org/10.1002/j.2158-1592.2008.tb00081.x

Wang, C., \& Ahmed, P. (2007). Dynamic capabilities: A review and research agenda. International Journal of Management Reviews, 9(1), 31-51. https://doi.org/10.1111/j.14682370.2007.00201.x

Yang, Y., Guo, L., Zhong, Z., \& Zhang, M. (2018). Selection of Technological Innovation for Service-Orientated Enterprises. Sustainability, 10(11), 1-13. https://doi.org/10.3390/su10113906

Zhang, M., \& Hartley, J. L. (2018). Guanxi, IT systems, and innovation capability: The moderating role of proactiveness. Journal of Business Research, 90, 75-86. https://doi.org/10.1016/j.jbusres.2018.04.036

Zhang, Y., Khan, U., Lee, S., \& Salik, M. (2019). The influence of management innovation and technological innovation on organization performance. a mediating role of sustainability. Sustainability (Switzerland), 11(2). https://doi.org/10.3390/su11020495 